\title{
Effect of Aether Field on Weak Deflection Angle of Black Holes
}

\author{
A. Övgün, ${ }^{1,2, *}$ İ. Sakalli, ${ }^{2, \dagger}$ and J. Saavedra ${ }^{1, \ddagger}$ \\ ${ }^{1}$ Instituto de Física, Pontificia Universidad Católica de Valparaíso, Casilla 4950, Valparaíso, Chile. \\ ${ }^{2}$ Physics Department, Faculty of Arts and Sciences, Eastern Mediterranean University, Famagusta, North Cyprus, via Mersin 10, Turkey.
} (Dated: August 12, 2019)

\begin{abstract}
We study the light rays in a static and spherically symmetric gravitational field of null aether theory (NAT). To this end, we employ the Gauss-Bonnet theorem to compute the deflection angle by a NAT black hole in the weak limit approximation. Using the optical metrics of the NAT black hole, we first obtain the Gaussian curvature and then calculate the leading terms of the deflection angle. Our calculations show how gravitational lensing is affected by the NAT field. We also show once again that the bending of light stems from a global and topological effect.
\end{abstract}

PACS numbers: 04.40.-b, 95.30.Sf, 98.62.Sb

Keywords: deflection of light; Gauss-Bonnet theorem; gravitational lensing; black hole; null aether theory

\section{INTRODUCTION}

According to the general relativity (GR) theory [1], gravity affects spacetime geometry, the gravitational field produced by matter can be powerful enough to drastically alter the ordinary causal structure of spacetime and produce a region, the so-called black hole $(\mathrm{BH})$, in which even light is held and no part can escape to infinity. For this reason, BHs have been studying in detail by scientists over the years.

The event horizon of a BH is a limit to which events cannot influence an observer on the opposite side. Namely, it is the gravitational point of no return that nobody can really "see" a BH. In fact, the event horizon is a null surface that separates the rays of light that reach infinity from those falling into singularity.On the other hand, astronomers can observe this curvature in spacetime as light from distant objects gets bent while going around foreground objects. In particular, if a $\mathrm{BH}$ is immersed in a bright region, like a disc of glowing gas, $\mathrm{BH}$ creates a dark region similar to a shadow [2,3]. This shadow, caused by the gravitational bending and capture of light by the event horizon, reveals a lot about the nature of these massive objects. From this information, the position and mass of the BH can be determined. For static and spherical symmetric spaces, suitable coordinate systems can be introduced to determine the location of the event horizon by looking at the places where the local light cones tilt over. This means that the existence of the event horizon (i.e., BH) is related to the local Lorentz invariance of time. Therefore, it is of great importance to study the properties of BHs in gravity theories that show local Lorentz invariance violations. Namely, in GR, the existence of BHs rides on the causal structure originated from both the Lorentz symmetry on matter fields a nd the local flatness th eorem. Therefore, it is na tural to examine whether BHs will still be formed in the absence of Lorentz symmetry or not; if they exits, it is even more important to analyze their physical features, since the signatures of Lorentz violations appear in the regime of strong gravity. On the other hand, when the Lorentz symmetry is broken and the causal structure is modified in a most radical way, $\mathrm{BH}$ solutions surprisingly do exist. In this new BH solutions, the event horizon is replaced by the universal horizon, which is to capture any mode independently of the propagation velocity $[4,5]$. Some BH solutions have been already found in restricted Lorentz violating gauges, such as spherical symmetry [4, 6] and slowly-rotating background, both in lower dimensions [7] and 4-dimensions [8-11]. Generally, numerical solutions to the equations of Hořava gravity and Einstein-aether theory admit those BHs. Nevertheless, there are rarely analytical solutions for this type of theories. However, all those solutions are obtained in the symmetry-restricted scenarios for various asymptotics [12]. In Einstein-aether theory, the vector field is timelike everywhere and explicitly breaks the boost sector of the Lorentz symmetry which has been studied extensively in the literature (see for instance [13]). In particular, aether models present themselves as phenomenological probes to test for the presence of Lorentz symmetry breaking (LSB) in astrophysical objects and cosmology. Aether models are nothing but a vector input to the Lagrangian density of the system having a nonvanishing vacuum expectation. Because of this property, the vector field dynamically selects a opted frame at each point in spacetime and automatically breaks the Lorentz invariance. This is a mechanism that reminds the breaking

\footnotetext{
*Electronic address: ali.ovgun@pucv.cl; URL: https : / / a ovgun . weebly • com

†Electronic address: izzet.sakalli@emu.edu.tr

${ }^{\ddagger}$ Electronic address: joel.saavedra@ucv.cl
} 
of local symmetry in Higgs mechanism [14] and serves as a phenomenological representation of the LSB terms in Standard Model Extension's gravitation sector [15].

NAT is one of the new vector-tensor theories of modified gravity theory [16]. In this theory, the dynamical vector field acts as the aether and exact spherically symmetric BH solution with charge is possible [17]. It was also discussed in [17] that NAT fields have effects on the solar system dynamics when extracting the Eddington-Robertson-Schiff parameters $\beta$ and $\gamma$, which appear in the perihelion precession and the light deflection expressions, for the NAT BHs. Furthermore, it was shown that at the post-Newtonian order although there is no contribution from the NAT field to the deflection of light rays passing near the BH (as in GR), however, NAT field gives contribution to the perihelion precession of planetary orbits. Later remark can play a role on solar system observations. The physical features (singularity structure, ADM mass, and thermodynamics) of the NAT BH are also analyzed in [17]. Moreover, NAT charge is able to reduce the horizon thermodynamics to that of the Reissner-Nordström-(A)dS BH of GR and modifies the circular orbits of massive and massless particles around the $\mathrm{BH}$. To utilize the differential deflection exhibited by weak lensing, first weak deflection angle is calculated. It depends on the mass distribution of the gravitational lensing system. Gibbons and Werner showed that it is possible to calculate the deflection angle in weak field limits using the Gauss-Bonnet theorem (GBT) and the optical geometry [18, 19]. In this method, they focus the domain outside the trajectory of light. Optical metric has geodesics, which are the spatial light rays, that the focusing of light rays are considered as a topological effect [20]. At the present time, the GW method has been applied to various spacetime metrics of black holes and wormholes (see for example [22-50] and references therein). We can write the domain surface define as $(D, \chi, g)$ using the the Euler characteristic $\chi$ and a Riemannian metric $g$. Then the GBT can be formulated as follows [18]:

$$
\iint_{D} K d S+\int_{\partial D} \kappa d t+\sum_{i} \alpha_{i}=2 \pi \chi(D)
$$

where, $\alpha_{i}$ is the exterior angle with $i^{\text {th }}$ vertex, Gaussian curvature, $K$ and geodesics curvature, $\kappa$. This method, only works for the asymptotically flat spacetimes and it is given by [18]:

$$
\hat{\alpha}=-\iint_{D} K d S
$$

In this article, our main purpose is to explore the NAT effects on the gravitational lensing. To this end, we organize the paper as follows: In section II, we briefly review the BH spacetime of the NAT. Section III is devoted to computation of the deflection angle by NAT BH using the GBT in weak field regime and in the plasma medium. We conclude our results in section IV. Natural units are used throughout this paper: $G=\hbar=c=1$.

\section{NAT BH SPACETIME}

The line-element of the asymptotically flat NAT BH is given by [17]

$$
d s^{2}=-h(r) d t^{2}+\frac{d r^{2}}{h(r)}+r^{2} d \theta^{2}+r^{2} \sin ^{2} \theta d \varphi^{2},
$$

where

$$
h(r)= \begin{cases}1-\frac{2 a_{1}^{2} b_{1}}{r^{1+q}}-\frac{2 a_{2}^{2} b_{2}}{r^{1-q}}-\frac{2 \tilde{m}}{r} & (\text { for } q \neq 0), \\ 1-\frac{2 m}{r} & (\text { for } q=0) .\end{cases}
$$

In the above equation, where $a_{1}, a_{2}, \tilde{m}$, and $m$ are just integration constants and

$$
q \equiv \sqrt{9+8 \frac{c_{1}}{c_{23}}}, \quad b_{1}=\frac{1}{8}\left[c_{3}-3 c_{2}+c_{23} q\right], \quad b_{2}=\frac{1}{8}\left[c_{3}-3 c_{2}-c_{23} q\right],
$$

in which $c_{1}, c_{2}, c_{3}$, and $c_{23}=c_{2}+c_{3}$ are the dimensionless constant parameters. Furthermore, the constants $\tilde{m}$ and $m$ are the mass parameters of the solutions.

As is obvious from Eq. (4), in the $q=0$ case, the metric is nothing but the well-known Schwarzschild spacetime, which is asymptotically flat. However, in the $q \neq 0$ case, to achieve asymptotically flat boundary conditions, one should consider the following cases separately (by definition $q>0$ [17]): 


$$
\left.h(r)\right|_{r \rightarrow \infty}=1 \begin{cases}\text { for } 0<q<1 & \left(\text { if } a_{1} \neq 0 \text { and } a_{2} \neq 0\right) \text { or }\left(\text { if } a_{1}=0 \text { or } b_{1}=0\right), \\ \text { for } 0<q & \left(\text { if } a_{2}=0 \text { or } b_{2}=0\right) .\end{cases}
$$

For the case $q=0$, the ADM mass is given by

$$
M_{A D M}=\frac{m}{G}
$$

On the other hand, for the case $q \neq 0$, one gets

$$
M_{A D M}=\left.\frac{1}{G}\left[\tilde{m}+(1+q) \frac{a_{1}^{2} b_{1}}{r^{q}}+(1-q) \frac{a_{2}^{2} b_{2}}{r^{-q}}\right]\right|_{r \rightarrow \infty} .
$$

Then one can also deduce from that above findings that to have asymptotically well-defined ADM mass for the NAT BHs:

$$
M_{A D M}=\frac{\tilde{m}}{G} \begin{cases}\text { for } 0<q<1 & \left(\text { if } a_{1}=0 \text { or } b_{1}=0\right) \\ \text { for } 0<q & \left(\text { if } a_{2}=0 \text { or } b_{2}=0\right)\end{cases}
$$

In this study, we shall consider the case of $a_{2}=0$. Then, the metric function $h(r)$ and the scalar aether field $\phi(r)$ take the following forms

$$
\begin{aligned}
& h(r)=1-\frac{2 a_{1}^{2} b_{1}}{r^{1+q}}-\frac{2 \tilde{m}}{r}, \\
& \phi(r)=\frac{a_{1}}{r^{(1+q) / 2}} .
\end{aligned}
$$

The location of the event horizon $r_{0}$ is given by $h\left(r_{0}\right)=0$ and the area of the event horizon is $A=4 \pi r_{0}^{2}$. Setting $a_{1}=G Q r_{0}^{(q-1) / 2}$, where $Q$ is the NAT "charge", Eqs. (10) and (11) become

$$
\begin{aligned}
& h(r)=1-\frac{2 G^{2} Q^{2} b_{1}}{r^{2}}\left(\frac{r_{0}}{r}\right)^{q-1}-\frac{2 \tilde{m}}{r}, \\
& \phi(r)=\frac{G Q}{r}\left(\frac{r_{0}}{r}\right)^{(q-1) / 2} .
\end{aligned}
$$

At the location of $r_{0}$, we have

$$
\begin{aligned}
& h\left(r_{0}\right)=1-\frac{2 G^{2} Q^{2} b_{1}}{r_{0}^{2}}-\frac{2 \tilde{m}}{r_{0}}=0, \\
& \phi\left(r_{0}\right)=\frac{G Q}{r_{0}} .
\end{aligned}
$$

It is worth noting that the horizon condition (14) is independent of the parameter $q$. In addition, the scalar aether field $\phi(r)$ resembles the electric potential at $r=r_{0}$.

Using the asymptotically flat solutions of NAT black hole given in [17],taking $q=1$, the metric function and scalar aether field is

$$
\begin{aligned}
& h(r)=1-\frac{2 a_{1}^{2} b_{1}}{r^{2}}-\frac{2 \tilde{m}}{r}, \\
& \phi(r)=\frac{a_{1}}{r^{1 / 2}} .
\end{aligned}
$$

The deflection angle of photon can be calculated using the following formula ( $r_{0}$ is the distance of closest approach) [56]:

$$
\hat{\alpha}\left(r_{0}\right)=-\pi+2 \int_{r_{0}}^{\infty} d r \frac{\sqrt{h(r)^{-1}}}{r \sqrt{\frac{r^{2}}{r_{0}^{2}} \frac{f\left(r_{0}\right)}{h(r)}-1}} .
$$

However, most cases it is not easy to solve this integral. For example in the case of $\frac{\tilde{m}}{r_{0}} \ll 1$, the deflection angle is found as too small, which is known as the weak lensing. Moreover, $\hat{\alpha}$ grows as $r_{0}$ closing to the photosphere until it diverges, and become the strong lensing. 


\section{CALCULATION OF WEAK DEFLECTION ANGLE OF NAT BH}

\section{A. Weak Deflection angle and GBT}

In this section, we calculate the weak deflection angle of NAT BH using the GBT. First, for simplicity, we assume that $\theta=\pi / 2$ for equatorial plane and use the spacetime metric given in 16 , to write the optical metric

$$
d t^{2}=\frac{d r^{2}}{\left(1-\frac{2 a_{1}^{2} b_{1}}{r^{2}}-\frac{2 \tilde{m}}{r}\right)^{2}}+\frac{r^{2}}{\left(1-\frac{2 a_{1}^{2} b_{1}}{r^{2}}-\frac{2 \tilde{m}}{r}\right)} d \phi^{2} .
$$

Then we calculate the Gaussian curvature of the optical NAT BH spacetime:

$$
K=\frac{R}{2} \approx-2 \frac{\tilde{m}}{r^{3}}+6 \frac{b_{1}(2 \tilde{m}-r) a_{1}^{2}}{r^{5}} .
$$

Here, using the above Gaussian curvature of the optical NAT BH spacetim in the GBT, we obtain the deflection angle. The GBT gives relation between the intrinsic geometry of the spacetime and its topology of the region $D_{R}$ in $M$, with boundary $\partial D_{R}=\gamma_{\tilde{g}} \cup C_{R}[18]$ :

$$
\int_{D_{R}} K \mathrm{~d} S+\oint_{\partial D_{R}} \kappa \mathrm{d} t+\sum_{i} \epsilon_{i}=2 \pi \chi\left(D_{R}\right) .
$$

Note that $\kappa$ is for the geodesic curvature $\kappa=\tilde{g}\left(\nabla_{\dot{\gamma}} \dot{\gamma}, \ddot{\gamma}\right)$, where $\tilde{g}(\dot{\gamma}, \dot{\gamma})=1$, and the unit acceleration vector $\ddot{\gamma}$, and $\epsilon_{i}$ corresponds to the exterior angle at the $i^{\text {th }}$ vertex. As $r \rightarrow \infty$, both jump angles reduces to $\pi / 2$, and it is found that $\theta_{O}+\theta_{S} \rightarrow \pi$. Because of $D_{R}$ is not singular, the Euler characteristic is $\chi\left(D_{R}\right)=1$. Hence, the GBT is

$$
\iint_{D_{R}} K \mathrm{~d} S+\oint_{\partial D_{R}} \kappa \mathrm{d} t+\theta_{i}=2 \pi \chi\left(D_{R}\right)
$$

in which $\gamma_{\tilde{g}}$ is a geodesic and $\theta_{i}=\pi$ denotes the total jump angle. Then we have $\kappa\left(\gamma_{\tilde{g}}\right)=0$. After recalling the Euler characteristic number, which is $\chi=1$, we find the remaining part that yields $\kappa\left(C_{R}\right)=\left|\nabla_{\dot{C}_{R}} \dot{C}_{R}\right|$ as $r \rightarrow \infty$. The radial component of the geodesic curvature is calculated as follows:

$$
\left(\nabla_{\dot{C}_{R}} \dot{C}_{R}\right)^{r}=\dot{C}_{R}^{\varphi} \partial_{\varphi} \dot{C}_{R}^{r}+\Gamma_{\varphi \varphi}^{r}\left(\dot{C}_{R}^{\varphi}\right)^{2}
$$

At very large $R, C_{R}:=r(\varphi)=r=$ const, we have

$$
\left(\nabla_{\dot{C}_{R}^{r}} \dot{C}_{R}^{r}\right)^{r} \rightarrow-\frac{1}{r}
$$

It is noted that the geodesic curvature does not depend on topological defects, $\kappa\left(C_{R}\right) \rightarrow r^{-1}$. Afterwards, from the optical spacetime metric (19), one can see that $\mathrm{d} t=r \mathrm{~d} \varphi$, and it follows:

$$
\kappa\left(C_{R}\right) \mathrm{d} t=\frac{1}{r} r \mathrm{~d} \varphi .
$$

Using the above results, the GBT equation reduces to this form:

$$
\iint_{D_{R}} K \mathrm{~d} S+\oint_{C_{R}} \kappa \mathrm{d} t \stackrel{r \rightarrow \infty}{=} \iint_{S_{\infty}} K \mathrm{~d} S+\int_{0}^{\pi+\hat{\alpha}} \mathrm{d} \varphi .
$$

In the weak deflection limit, one may assume that the light ray is given by $r(t)=u / \sin \varphi$ at zeroth order. Then we use straight line approximation [18] as $r=u / \sin \phi$, where $u$ is the impact parameter, and Eq. (2) becomes:

$$
\hat{\alpha}=-\int_{0}^{\pi} \int_{\frac{u}{\sin \phi}}^{\infty} K d S
$$


where $d S=r d r d \phi$. Note that we ignore the higher order terms. Hence, Eqs. (20) and (27) are simplified to the following expression for the deflection angle of NAT BH in second order due to the weak lensing:

$$
\hat{\alpha} \simeq 3 / 2 \frac{a_{1}^{2} b_{1} \pi}{u^{2}}+4 \frac{\tilde{m}}{u},
$$

where the deflection angle is full agreement with the equation (115) in the paper [17]. Here, one realizes that depending on the sign of the aether field parameter $b_{1}$, the light deflection can be more or less than the GR value given by the above first term. For $b_{1}<0$, the aether field decreases the light deflection angle relative to the Schwarzschild case in GR. This is similar to the effect of charge in the Reissner-Nordström solution $[57,58]$ for the weak-field limits. Thus, in the presence of $b_{1}>0$ NAT parameter, the aether increases the deflection angle, and deflection angle reduces to case of Schwarzschild $\mathrm{BH}$ when $b_{1}=0$. The deflection angle in the leading order terms is seen to be in agreement with [17].

\section{B. Weak Deflection angle of NAT BH in a plasma medium}

To take in account of the effects of plasma [33], in this subsection we shall use the case in which light travels from vacuum to a hot, ionized gas medium. Let $v$ be the velocity of light through the plasma. Then, the refractive index $n(r)$ is written as follows:

$$
n(r) \equiv \frac{c}{v}=\frac{1}{d r / d t} \quad\{\because c=1\} .
$$

Afterwards, we obtain the refractive index $n(r)$ for an NAT BH: [33],

$$
n(r)=\sqrt{1-\frac{\omega_{e}^{2}}{\omega_{\infty}^{2}}\left(1-\frac{2 a_{1}^{2} b_{1}}{r^{2}}-\frac{2 \tilde{m}}{r}\right)},
$$

where $\omega_{e}$ and $\omega_{\infty}$ are the electron plasma frequency and the photon frequency measured by an observer at infinity, respectively. The line-element (16) can be rewritten as:

$$
d \sigma^{2}=g_{i j}^{\mathrm{opt}} d x^{i} d x^{j}=\frac{n^{2}(r)}{1-\frac{2 a_{1}^{2} b_{1}}{r^{2}}-\frac{2 \tilde{m}}{r}}\left[\frac{d r^{2}}{1-\frac{2 a_{1}^{2} b_{1}}{r^{2}}-\frac{2 \tilde{m}}{r}}+r^{2} d \phi^{2}\right] .
$$

The optical Gaussian curvature becomes:

$$
K \approx-2 \frac{\tilde{m}}{r^{3}}-3 \frac{\tilde{m} \omega_{e}^{2}}{\omega_{\infty}^{2} r^{3}}+\left(-6 r^{-4}+12 \frac{\tilde{m}}{r^{5}}+\left(-10 \frac{1}{\omega_{\infty}^{2} r^{4}}+52 \frac{\tilde{m}}{\omega_{\infty}^{2} r^{5}}\right) \omega_{e}^{2}\right) a_{1}^{2} b_{1} .
$$

On the other hand, it follows from Eq. (31)

$$
\left.\frac{d \sigma}{d \varphi}\right|_{C_{r}}=\sqrt{1-\frac{\omega_{e}^{2}}{\omega_{\infty}^{2}}\left(1-\frac{2 a_{1}^{2} b_{1}}{r^{2}}-\frac{2 \tilde{m}}{r}\right)}\left(\frac{r^{2}}{1-\frac{2 a_{1}^{2} b_{1}}{r^{2}}-\frac{2 \tilde{m}}{r}}\right)^{1 / 2},
$$

that we have

$$
\left.\lim _{R \rightarrow \infty} \kappa_{g} \frac{d \sigma}{d \varphi}\right|_{C_{R}}=1
$$

For the limit of $R \rightarrow \infty$, and using the straight light approximation $r=u / \sin \varphi$, the GBT becomes [33]:

$$
\left.\lim _{R \rightarrow \infty} \int_{0}^{\pi+\alpha}\left[\kappa_{g} \frac{d \sigma}{d \varphi}\right]\right|_{C_{R}} d \varphi=\pi-\lim _{R \rightarrow \infty} \int_{0}^{\pi} \int_{\frac{u}{\sin \varphi}}^{R} \mathcal{K} d S .
$$


Consequently, the deflection angle yields

$$
\hat{\alpha} \approx 6 \frac{\tilde{m} \omega_{e}^{2}}{u \omega_{\infty}^{2}}+4 \frac{\tilde{m}}{u}+\frac{5 a_{1}^{2} b_{1} \omega_{e}^{2} \pi}{2 u^{2} \omega_{\infty}^{2}}+\frac{3 a^{2} b_{1} \pi}{2 u^{2}},
$$

where the photon rays are moving in a medium of homogeneous plasma. Note that absence of plasma $\left(\omega_{e}=0\right)$, or $\left(\omega_{e} / \omega_{\infty} \rightarrow 0\right)$ this deflection angle reduces to the vacuum case calculated in 28 . It is clear that for the photons propagating in a homogeneous plasma for the case of frequency $\omega_{e} / \omega_{\infty}=6 \times 10^{-3}$ [59], the deflection angle is increased. But, the effect of the plasma medium can not be detected easily due to its small value in near future observations.

\section{CONCLUSIONS}

In this study, we have studied weak gravitational lensing of NUT BH, which is a solution of the new vectortensor theory. After integrating the deflection angle integral (27), analytically, we have shown that if $\frac{\tilde{m}}{r_{0}} \ll 1$, the deflection angle gets too small. The latter remark is the evidence of the weak lensing. Remarkably, $\hat{\alpha}$ increases as $r_{0}$ approaches the photosphere until it diverges to produce the strong lensing. The aether field parameter $b_{1}$ modifies the gravitational lensing in such a way that when $b_{1}<0$, the aether field decreases the light deflection angle relative to the Schwarzschild BH of GR. This result is analogue to the effect of charge in the Reissner-Nordström BH $[57,58]$ in the weak-field limit. On the other hand, positive NAT parameter increases the deflection angle that reduces to case of Schwarzschild BH when $b_{1}=0$. Besides, in the existence of plasma $\left(\omega_{e}=0\right)$, the photons propagate in a homogeneous plasma for the case of frequency $\omega_{e} / \omega_{\infty}=6 \times 10^{-3}$ [59] and the deflection angle gets increased. But, the effect of the plasma medium seems not be detected in the near future due its infinitesimal value [60].

As a future work, we plan to proceed to study our topological frame work on the gravitational lensing of rotating NAT BHs, which can be obtained through Newman-Janis algorithm [61], as same as Kerr and/or BTZ which are accepted as more realistic $\mathrm{BH}$ geometries. Spraying the particles from their ergosphere effects the moving photons around the rotating BH's photon sphere. Therefore, it will be interesting to analyze the deflection angle of the rotating NAT BH. We believe that the results to be obtained will shed light on future observations.

\section{Acknowledgments}

This work was supported by Comisión Nacional de Ciencias y Tecnología of Chile through FONDECYT Grant $N^{\circ}$ 3170035 (A. Ö.).

[1] K. Schwarzschild, "On the gravitational field of a mass point according to Einstein's theory," Sitzungsber. Preuss. Akad. Wiss. Berlin (Math. Phys. ) 1916, 189 (1916).

[2] S. E. Gralla, D. E. Holz and R. M. Wald, “Black Hole Shadows, Photon Rings, and Lensing Rings," Phys. Rev. D 100, 024018 (2019).

[3] M. Safarzadeh, A. Loeb and M. Reid, “Constraining a black hole companion for M87* through imaging by the Event Horizon Telescope," Mon. Not. Roy. Astron. Soc. 488, L90 (2019).

[4] E. Barausse, T. Jacobson and T. P. Sotiriou, "Black holes in Einstein-aether and Horava-Lifshitz gravity," Phys. Rev. D 83, 124043 (2011).

[5] D. Blas and S. Sibiryakov, "Horava gravity versus thermodynamics: The Black hole case," Phys. Rev. D 84, 124043 (2011).

[6] C. Eling and T. Jacobson, "Black Holes in Einstein-Aether Theory," Class. Quant. Grav. 23, 5643 (2006) Erratum: [Class. Quant. Grav. 27, 049802 (2010)].

[7] T. P. Sotiriou, I. Vega and D. Vernieri, “Rotating black holes in three-dimensional Horava gravity," Phys. Rev. D 90, 044046 (2014).

[8] E. Barausse and T. P. Sotiriou, "A no-go theorem for slowly rotating black holes in Horava-Lifshitz gravity," Phys. Rev. Lett. 109, 181101 (2012) Erratum: [Phys. Rev. Lett. 110, 039902 (2013)].

[9] E. Barausse and T. P. Sotiriou, "Slowly rotating black holes in Horava-Lifshitz gravity," Phys. Rev. D 87, 087504 (2013).

[10] E. Barausse and T. P. Sotiriou, "Black holes in Lorentz-violating gravity theories," Class. Quant. Grav. 30, 244010 (2013).

[11] A. O. Barvinsky, D. Blas, M. Herrero-Valea, S. M. Sibiryakov and C. F. Steinwachs, "Renormalization of Horava gravity," Phys. Rev. D 93, 064022 (2016).

[12] J. Bhattacharyya and D. Mattingly, “Universal horizons in maximally symmetric spaces,” Int. J. Mod. Phys. D 23, 1443005 (2014). 
[13] C. Eling and T. Jacobson, "Spherical solutions in Einstein-aether theory: Static aether and stars," Class. Quant. Grav. 23, 5625 (2006) Erratum: [Class. Quant. Grav. 27, 049801 (2010)].

[14] D. Griffiths, Introduction to Elementary Particles, 2nd, Revised Edition(John Wiley and Sons, Mörlenbach, 2008).

[15] G. M. Pelaggi, A. D. Plascencia, A. Salvio, F. Sannino, J. Smirnov and A. Strumia, “Asymptotically Safe Standard Model Extensions?," Phys. Rev. D 97, 095013 (2018).

[16] M. Gürses and Ç. Şentürk, “A Modified Gravity Theory: Null Aether," Commun. Theor. Phys. 71, 312 (2019).

[17] M. Gurses, Y. Heydarzade and C. Senturk, "NAT Black Holes," arXiv:1907.09584 [gr-qc].

[18] G. W. Gibbons and M. C. Werner, "Applications of the Gauss-Bonnet theorem to gravitational lensing," Class. Quant. Grav. 25, 235009 (2008)

[19] M. C. Werner, “Gravitational lensing in the Kerr-Randers optical geometry,” Gen. Rel. Grav. 44, 3047 (2012)

[20] A. Övgün, "Weak field deflection angle by regular black holes with cosmic strings using the Gauss-Bonnet theorem," Phys. Rev. D 99, 104075 (2019)

[21] A. Ishihara, Y. Suzuki, T. Ono, T. Kitamura and H. Asada, “Gravitational bending angle of light for finite distance and the Gauss-Bonnet theorem," Phys. Rev. D 94, 084015 (2016).

[22] H. Arakida, "Light deflection and GaussBonnet theorem: definition of total deflection angle and its applications," Gen. Rel. Grav. 50, 48 (2018).

[23] T. Ono, A. Ishihara and H. Asada, "Deflection angle of light for an observer and source at finite distance from a rotating wormhole," Phys. Rev. D 98, 044047 (2018).

[24] T. Ono, A. Ishihara and H. Asada, "Gravitomagnetic bending angle of light with finite-distance corrections in stationary axisymmetric spacetimes," Phys. Rev. D 96, 104037 (2017).

[25] K. Jusufi, A. Övgün and A. Banerjee, "Light deflection by charged wormholes in Einstein-Maxwell-dilaton theory," Phys. Rev. D 96, n084036 (2017) Addendum: [Phys. Rev. D 96, 089904 (2017)].

[26] A. Övgün, K. Jusufi and I. Sakalli, "Exact traversable wormhole solution in bumblebee gravity," Phys. Rev. D 99, 024042 (2019).

[27] K. Jusufi and A. Övgün, “Gravitational Lensing by Rotating Wormholes,” Phys. Rev. D 97, 024042 (2018).

[28] K. Jusufi and A. Övgün, "Light Deflection by a Quantum Improved Kerr Black Hole Pierced by a Cosmic String," Int. J. Geom. Meth. Mod. Phys. (2019) 1950116.

[29] K. Jusufi, M. C. Werner, A. Banerjee, and A. Övgün, “Light Deflection by a Rotating Global Monopole Spacetime," Phys. Rev. D 95, no. 10, 104012 (2017).

[30] K. Jusufi, I. Sakalli, and A. Övgün, "Effect of Lorentz Symmetry Breaking on the Deflection of Light in a Cosmic String Spacetime," Phys. Rev. D 96, no. 2, 024040 (2017).

[31] T. Ono, A. Ishihara, and H. Asada, "Deflection angle of light for an observer and source at finite distance from a rotating global monopole," Phys. Rev. D 99, no. 12, 124030 (2019).

[32] K. Jusufi, A. Övgün, A. Banerjee and I. Sakalli, "Gravitational lensing by wormholes supported by electromagnetic, scalar, and quantum effects," arXiv:1802.07680 [gr-qc]. Accepted from (EPJ Plus).

[33] G. Crisnejo and E. Gallo, "Weak lensing in a plasma medium and gravitational deflection of massive particles using the Gauss-Bonnet theorem. A unified treatment," Phys. Rev. D 97, 124016 (2018)

[34] G. Crisnejo, E. Gallo, and A. Rogers, "Finite distance corrections to the light deflection in a gravitational field with a plasma medium," Phys. Rev. D 99, 124001 (2019).

[35] G. Crisnejo, E. Gallo, and J. R. Villanueva, "Gravitational lensing in dispersive media and deflection angle of charged massive particles in terms of curvature scalars and energy-momentum tensor," arXiv:1905.02125 [gr-qc].

[36] I. Sakalli and A. Ovgun, "Hawking Radiation and Deflection of Light from Rindler Modified Schwarzschild Black Hole," EPL 118, no. 6, 60006 (2017).

[37] A. Övgün, G. Gyulchev, and K. Jusufi, “Weak Gravitational lensing by phantom black holes and phantom wormholes using the Gauss-Bonnet theorem," Annals Phys. 406, 152 (2019).

[38] K. Jusufi and A. Övgün, "Effect of the cosmological constant on the deflection angle by a rotating cosmic string," Phys. Rev. D 97, 064030 (2018).

[39] K. Jusufi, A. Övgün, J. Saavedra, Y. Vasquez, and P. A. Gonzalez, “Deflection of light by rotating regular black holes using the Gauss-Bonnet theorem," Phys. Rev. D 97, 124024 (2018).

[40] A. Övgün, “Light deflection by Damour-Solodukhin wormholes and Gauss-Bonnet theorem," Phys. Rev. D 98, 044033 (2018).

[41] A. Övgün, K. Jusufi, and I. Sakalli, "Gravitational lensing under the effect of Weyl and bumblebee gravities: Applications of GaussBonnet theorem," Annals Phys. 399, 193 (2018).

[42] A. Övgün, "Deflection angle of photon through dark matter by black holes and wormholes using the Gauss-Bonnet theorem," Universe 5, 115 (2019).

[43] A. Övgün, I. Sakalli, and J. Saavedra, "Weak gravitational lensing by Kerr-MOG Black Hole and Gauss-Bonnet theorem," arXiv:1806.06453 [gr-qc] (Accepted from AOP).

[44] A. Övgün, I. Sakalli, and J. Saavedra, "Shadow cast and Deflection angle of Kerr-Newman-Kasuya spacetime," JCAP 1810, 041 (2018).

[45] W. Javed, R. Babar, and A. Övgün, "The effect of the Brane-Dicke coupling parameter on weak gravitational lensing by wormholes and naked singularities," Phys. Rev. D 99, 084012 (2019).

[46] W. Javed, R. Babar, and A. Övgün, "Effect of the Dilaton Field on Deflection Angle of Massive Photons by Black Holes in Einstein-Maxwell-Dilaton-Axion," Theory. Preprints 2019050148, 2019. (doi: 10.20944/preprints201905.0148.v1)

[47] W. Javed, J. Abbas, and A. Övgün, " Deflection Angle of Photon from Magnetized Black Hole and Effect of Nonlinear Electrodynamics," Preprints 2019, 2019030260 (doi: 10.20944/preprints201903.0260.v1). (Accepted from EPJC). 
[48] W. Javed, J. Abbas and A. Övgün, "Effect of the Quintessential Dark Energy on Weak Deflection Angle by Kerr-Newmann Black Hole," Preprints 2019, 2019060124. (doi: 10.20944/preprints201906.0124.v1).

[49] W. Javed, j. Abbas and A. Övgün, "Effect of the Hair on Deflection Angle by Asymptotically Flat Black Holes in EinsteinMaxwell-Dilaton Theory," Preprints 2019, 2019060101 (doi: 10.20944/preprints201906.0101.v1).

[50] Y. Kumaran and A. Övgün, “Weak Deflection Angle of Extended Uncertainty Principle Black Holes," arXiv:1905.11710 [grqc].

[51] S. W. Hawking, "Black hole explosions," Nature 248, 30 (1974).

[52] S. W. Hawking, "Particle Creation by Black Holes," Commun. Math. Phys. 43, 199 (1975) Erratum: [Commun. Math. Phys. 46, 206 (1976)].

[53] R. J. Adler, P. Chen, and D. I. Santiago, “The Generalized uncertainty principle and black hole remnants,” Gen. Rel. Grav. 33, 2101 (2001).

[54] J. R. Mureika, “Extended Uncertainty Principle Black Holes,” Phys. Lett. B 789, 88 (2019).

[55] B. Bolen and M. Cavaglia, "(Anti-)de Sitter black hole thermodynamics and the generalized uncertainty principle," Gen. Rel. Grav. 37, 1255 (2005.

[56] K. S. Virbhadra, D. Narasimha, and S. M. Chitre, “Role of the scalar field in gravitational lensing," Astron. Astrophys. 337, 1 (1998).

[57] Ya-Peng Hu, H. Zhang, J-Peng Hou, L-Zun Tang, "Perihelion precession and deflection of light in the general spherically symmetric spacetime," Adv. High Energy Phys. 2014, 604321 (2014).

[58] P. Amore and S. Arceo, "Analytical formulas for gravitational lensing," Phys. Rev. D 73, 083004 (2006).

[59] G. S. Bisnovatyi-Kogan and O. Y. Tsupko, “Gravitational lensing in a non-uniform plasma," Mon. Not. Roy. Astron. Soc. 404, 1790 (2010).

[60] K. Parfrey, A. Philippov and B. Cerutti, “First-Principles Plasma Simulations of Black-Hole Jet Launching,” Phys. Rev. Lett. 122, 035101 (2019).

[61] R. Shaikh, "Black hole shadow in a general rotating spacetime obtained through Newman-Janis algorithm," Phys. Rev. D 100, 024028 (2019). 